\title{
RAPID APPLICATION DEVELOPMENT USING WEB TECHNOLOGIES An Application to Communicative Competence Promotion of Children with ASD
}

\author{
Margarida Lucas da Silva \\ Instituto Superior Técnico - UTL, Lisboa, Portugal \\ marga.lucas.silva@ist.utl.pt \\ Carla Simões \\ Escola Superior de Saúde de Setúbal - IPS, Setúbal, Portugal \\ Faculdade de Ciências Sociais e Humanas - UNL, Lisboa, Portugal \\ m.carla.simoes@gmail.com \\ Hugo Plácido da Silva \\ Instituto Superior Técnico - UTL, Lisboa, Portugal \\ hugo.silva@lx.it.pt \\ Tiago Guerreiro \\ Instituto Superior Técnico - UTL/INESC-ID, Lisboa, Portugal \\ tjvg@vimmi.inesc-id.pt \\ Fernanda Botelho \\ Escola Superior de Saúde de Setúbal - IPS, Setúbal, Portugal \\ fernanda.botelho@ese.ips.pt
}

Keywords: $\quad$ RAD; Autism Spectrum Disorders; Communicative Competence; Usability; Web Technologies.

Abstract: This paper describes a framework, devised for rapid application development, and rapid application customization of standalone multimedia and rich content software, targeted at students with special needs. We present a proof-of-concept software application for promotion and training of social communication skills in children with autism spectrum disorders. Web technologies and related standards are used as a way of easily involving tutors, professionals in the field, and end users themselves in the software development and customization process with minimal training and support.

\section{INTRODUCTION}

Continuous technical evolution in computer programming has provided different abstraction levels, gradually empowering a broader community, which does not necessarily include technologically proficient individuals, with tools for software development (Hasse and Guy, 2007) (Martin, 1991). Groundbreaking examples of this trend are the Wikibased tools for website construction (Barrett, 2008).

Although this has led to increasingly userfriendly software applications, the target is mostly the general population (Unger and Chandler, 2009).
For people with special needs, the learning curves are highly user dependant, and even contents may need to be adapted according to their specific needs (Emiliani et al., 2009) (Helal et al., 2008).

Children with autism spectrum disorders (ASD) are one such group; The difficulties of integration and learning in special education schools, has led to guidelines for integration of children with ASD into regular education schools (UNESCO, 1994). Computer aided tools play a major role in the development of adequate educational responses in this group, given the significant limitations to the level of activity and participation that arise due to 
permanent and continued difficulties in communication, learning, mobility, autonomy, interpersonal relationships and social participation.

Standard software applications are fitted with functions and graphical stimuli that may lead to distraction, dissatisfaction or frustration. In general, tools with a high level of customization are required (Boujarwah et al., 2010) (Putman, 2008).

Existing software tools applicable to training of children with ASD, are generally targeted at cognitive or communication enhancement skills (Sensory Software, 2010) (People CD, 2008). These either focus more on the delivery of rich multimedia content, and less on the customization capabilities, or vice versa.

It is still difficult for tutors, caregivers, and other professionals in the field, to become autonomous in the process of managing and adapting the available tools to the needs of children with ASD. Web technologies and related standards have provided several well-supported means, which can be easily mastered by a broad audience. These technologies can be extended to application development, promoting the involvement of the "user in the loop" due to the simplicity they provide.

This paper describes a standalone software application for promotion and training of social communication skills in children with ASD. It is based on a framework for rapid application development (RAD), and rapid application customization (RAC). The underlying framework can be generalized for the development of software targeted at people with special needs, that tutors and professionals in the field can fully manipulate autonomously with little training and support.

The paper is organized as follows: Section 2 goes through the RAD/RAC framework depicting how we achieve the desired flexibility and adaptability; Section 3 describes the software application for communication skills training in children with ASD while in Section 4 we present the experimental evaluation performed with the target users; and finally, Section 5 summarizes the main results and conclusions.

\section{FRAMEWORK}

The framework was designed with both the endusers and the people behind application management in mind, targeting the seamless delivery of rich multimedia content, while providing high customization levels, easy content management and maintenance by non-technologically proficient individuals.

We found the answers to these requirements in web technologies. Web browsers are currently the best platforms for rich multimedia content delivery, and web standards provide some of the best tools for GUI layout and formatting. Figure 1 illustrates the proposed framework, where the whole application structure is supported around a web browser.

The presentation layer takes advantage of the rendering capabilities, the logic layer relies on the scripting capabilities, and the data/persistence layer is supported on the capabilities of interoperability between the browser and the OS. Furthermore, modern web browsers have a set of plug-ins that extends their multimedia capabilities.

Figure 1 depicts this architecture, which presents interesting properties for RAD/RAC of full-featured standalone multimedia applications: (a) no special development tools are required; (b) the code is interpreted rather than compiled; (c) changes to the screen structure and navigation are straightforward; and (d) content management can be performed in a way that is familiar to most users.

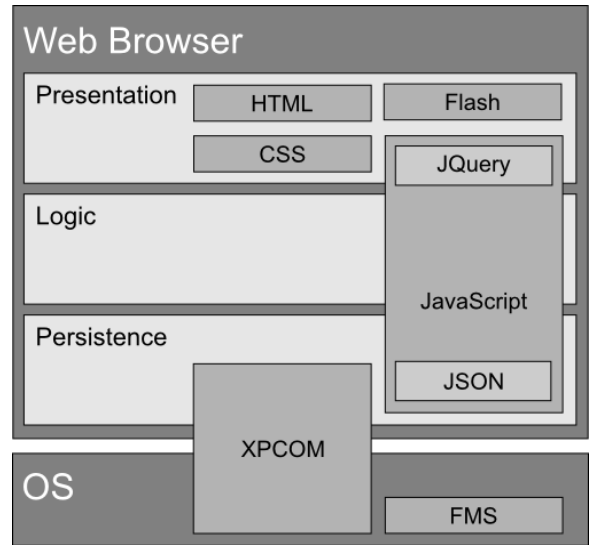

Figure 1: Architecture of the proposed framework.

\section{APPLICATION}

Interpersonal communication and interaction skills of children with ASD may be very limited, which creates the need to develop a tool to support their needs for integration in social life. This is important not only for educational purposes, but also for leisure, so that individuals can share their tastes with others, comment on existing contents, and communicate with their peers. Furthermore, tutors 
have the need to setup contents adapted to the reality of each group, to stimulate them and promote greater autonomy in normal processes such as viewing photos, listening to music, among other activities.

In this context, we developed a tool directed to the specifics of this topic. It has the twofold objective of encouraging socialization facilitating interaction among children with ASD, while allowing tutors to easily customize the contents and look-and-feel of the tool because each child is different, presenting divergent capabilities, habits and needs.

The application can be fully customized with icons, background images and the contents that allow each child to be more effective while using it. Children can even use their own drawings or favourite images to better associate each feature of the software with the underlying function.

Tutors have full control over the software and they can intervene virtually at all levels. They can act at the content level, where contents are structured and organized under the application folder tree, just as any other document is organized in regular computer work, as we can see in figure 2. But they can also act all the way up to the presentation level, where they are able to manage the screen and navigation structure by simply editing the HTML files.

$$
\begin{aligned}
& \square \text { audio } \\
& \square \text { images } \\
& \square \text { stories } \\
& \square \text { video }
\end{aligned}
$$

Figure 2: Example of a content folder tree.

All the activities are targeted at communication skills training. They are based in multimedia content: images, videos, audio, and stories that children can autonomously browse through and learn from. In addition there is also a message board, and content preference sharing options, these being the main contribution of the tool to the field, when compared to existing work.

In the message board children can post messages to their colleagues. The sender child has a buddy list with names and photos of all counterparts, from which the receiver colleague can be chosen. The message can then be written either through text, picture exchange (Bondy, 1994), or a combination of both methods. When the receiver colleague opens the message board, he will immediately be able to see all of the messages sent to him by others. Figure 2 shows the message board screen.

For content preference sharing, associated to each multimedia element (image, sound or video), there is a positive and a negative preference option. These allow the child to express and share with its counterparts at any time, his/her preference regarding the individual element. This information is associated with the content and shown every time it is accessed, allowing all children to see the opinion of their colleagues. Figure 2 illustrates one of the activities with the content preference-sharing pane on the right area of the screen.

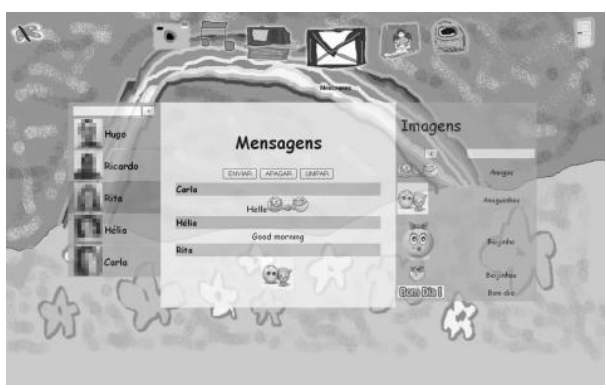

Figure 2: Message board of the application.

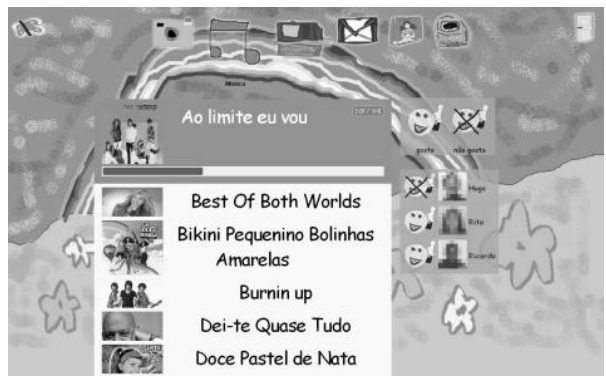

Figure 3: Activity with content preference-sharing pane.

\section{EXPERIMENTAL RESULTS}

The application was tested with three children with ASD, attending a regular school and integrated in a "unit of structured teaching". Experiments were conducted with the supervision of their tutor that, over a period of 12 weeks, performed the tests and collected evidence based on observation, which was used to evaluate their behaviour before, throughout, and after the platform being put to use.

Due to their several cognitive impairments, other two children were not eligible to participate. For initial customization of the software, the tutor listed the different activities and asked one of the children to draw images that, for her, would be more meaningful of the underlying concept.

As a result, the children themselves created the look-and-feel of the UI. By introducing contents 
generated by their counterparts, children recognize and develop a feeling of belong towards the software, which works as an additional drive towards its use. Starting with an empty working base, the tutor was able to autonomously customize and fine-tune the application contents within a 2week period.

Results with the children over a 3-month period have shown that through the message board tutors were able to stimulate the interest and need of children's communication. When using the software, children would first browse through the message board to read and reply to messages sent to them by their tutors and by their counterparts.

There are some evidences that point up that this tool can also be used to promote reading and writing competences, through the interpretation of written messages, and the stories' retell (children can access Photostory from the framework for this purpose). It was also observed that the content preference sharing options brought the children together over common preferences through the software. The opinion of their counterparts would drive each child to access and also share their preference for a given content in the application.

From the test period, preliminary results point to the fact that children exhibit increased motivation and interaction. Two of the subjects actually did not interact at all with one another, and through the implementation of methodology supported by the software application they started to tolerate one another and co-operate in the activities provided by the software.

\section{CONCLUSIONS}

In this paper we described a standalone software application for promotion and training of communication skills in children with ASD. The application is based on a RAD/RAC framework that can be generalized for the development of other software tools targeted at people with other special needs in a "user in the loop" approach.

Our goal is to provide non-technologically proficient users with the tools to manage the content and customize the application. With the proposed methodology even the users themselves can participate in the customization process by creating icons, background images and symbols that better reflect the underlying concepts to their eyes.

The application was tested in a real world scenario. Experimental evaluation has shown that, within a 2-week period, the tutor was able to autonomously fine-tune the tool and customize the contents. Afterwards, the tool was implemented in the daily routine of the children where it was in use during a 3-month period.

Results have shown the effectiveness of the proposed framework and application. Tutors are able to autonomously manage the contents and customize the application, according to the children's attention and response to contents. Children demonstrated higher motivation and increased interaction patterns.

Future work will focus on portability and community building, allowing the framework to be used in mobile platforms and extending its local capabilities with services made available by the cloud.

\section{REFERENCES}

Barrett, D., 2008. Mediawiki (Wikipedia and Beyond), O'Reilly Media.

Bondy A., Frost L., 1994. The Picture Exchange Communication System. Focus on Autism and Other Developmental Disabilities, Vol. 9, No. 3, 1-19.

Boujarwah, F., Hong, H., Isbell, J., Arriaga, R., Abowd, G., 2010. Training Social Problem Solving Skills in Adolescents with High-Functioning Autism. In Proceedings of the 4th International Conference on Pervasive Computing Technologies for Healthcare.

Emiliani, P., Burzagli. L., Como, A., Gabbanini, F., Salminen, A., 2009. Assistive Technology from Adapted Equipment to Inclusive Environments. AAATE 2009, Volume 25 Assistive Technology Research Series, IOS Press.

Haase, C., Guy, R., 2007. Filthy Rich Clients: Developing Animated and Graphical Effects for Desktop Java Applications, Addison Wesley.

Helal, A., Mokhtari, M., Abdulrazak, B.. 2008. The Engineering Handbook of Smart Technology for Aging, Disability and Independence, Wiley.

Martin, J., 1991. Rapid Application Development, Macmillan Publishing Co., Inc.

People CD, 2008. Zac Browser, The First Internet Browser Developed Specifically for Children Living with Variants of Autism Spectrum Disorders. Cited in 2010/07/01: http://www.zacbrowser.com/.

Putman, C., Chong, L., 2008. Software and Technologies Designed for People with Autism: What do Users Want?. In Proceedings of ASSETS 2008.

Sensory Software, 2010. The Grid 2, an all-in-one package for communication and access. Cited in 2010/07/01: http://www.sensorysoftware.com/thegrid2.html.

UNESCO, 1994. The Salamanca Statement and Framework for Action on Special Needs Education, UNESCO (ED-94/WS/18).

Unger, R., Chandler, C., 2009. A Project Guide to UX Design: For user experience designers in the field or in the making, New Riders Press. 